Trauma Berufskrankh 2009 · 11[Suppl 1]:49-54 DOI 10.1007/s10039-008-1461-2

Online publiziert: 5. Dezember 2008

c) Springer Medizin Verlag 2008
N.M. Meenen ${ }^{1} \cdot$ J.-P. Petersen ${ }^{2} \cdot$ P. Ueblacker ${ }^{3}$

${ }^{1}$ Pädiatrische Sportmedizin, Kinderorthopädie, Altonaer Kinderkrankenhaus, Hamburg

2 Unfall- Hand- und Wiederherstellungschirurgie, Universitätsklinikum Hamburg Eppendorf, Hamburg

${ }^{3}$ Müller-Wohlfahrt Centrum für Orthopädie und Sportmedizin, München

\section{Osteochondrale Transplantation}

\section{Ein bewährtes Therapieverfahren für größere Knorpeldefekte an Knie und OSG}

$\mathrm{Zu}$ umschriebenen Knorpeldefekten bei jungen Menschen kommt es infolge nicht sachgerechter sportlicher Betätigung, durch Unfall oder vergleichbare Überlastung. Auch Veränderungen der subchondralen Biomechanik oder Perfusion können nachhaltig auf die Gelenkflächenintegrität einwirken. Die Tendenz zur spontanen Heilung selbst solcher lokalisierter Knorpelschäden ist sehr gering, die klinische Symptomatik so eindrucksvoll, dass der englische Chirurg W. Hunter bereits 1743 konstatierte:

„Wenn wir bei allen bisherigen Chirurgen bis zur heutigen Zeit nachlesen, stellen wir fest: Knorpelschäden sind sehr unangenehm, sie heilen nie. Defekter Knorpel ist schwieriger zu behandeln als Knochenschäden."

Wir konstatieren heute, nach mehr als 250 Jahren, dass diese grundsätzliche Aussage weitgehend Bestand hat.

In der Vergangenheit wurden sehr viele Arten der Behandlung von Knorpelschäden vorgestellt, mit welchen auch mehr oder weniger erfolgreich die typische Beschwerden wie Schmerz, Blockierungen, Gelenkergüsse und Bewegungseinschränkungen bekämpft werden konnten. Ein allgemein und umfassend erfolgreiches Konzept, das zu einer vollständigen Ausheilung der Defekte mit uneingeschränkter Wiederaufnahme der sportlichen oder alltäglichen Betätigungen führt, existiert noch nicht. Daher werden im Folgenden die möglichen Konzepte und der Beitrag, den sie zur Lösung des Problems Knorpelschaden beitragen können, besprochen.

Ursachen für Gelenkknorpeldefekte. Neben dem primären Verschleiß und der Ostoechondrosis dissecans spielen insbesondere bei jüngeren Patienten zunehmend Unfälle auch beim Sport eine entscheidende Rolle. Die entsprechenden Traumen treten v. a. in den stark beanspruchten Gelenken der unteren Extremitäten auf. Hier stehen das Kniegelenk und das obere Sprunggelenk im Vordergrund. Häufig handelt es sich primär nicht um isolierte Knorpelschäden, sondern um Folgen einer komplexen Gelenkverletzung. So sind $20-40 \%$ der vorderen Kreuzbandverletzungen durch die resultierende Instabilität oder begleitende Meniskusschäden mit Knorpelläsionen vergesellschaftet.

Spontanheilung. Der hyaline Knorpel hat nur eine sehr geringe Tendenz zur Selbstheilung. Oberflächliche Knorpelläsionen heilen aufgrund der fehlenden Durchblutung gar nicht und tiefere Läsionen, welche bis in den durchbluteten subchondralen Knochen reichen, werden spontan lediglich mit dem biomechanisch und biochemisch minderwertigen Faserknorpel gefüllt.

\section{Beurteilung der Knorpelschäden}

Erfolgreiche Konzepte zur Behandlung von Knorpelschäden setzen eine klinische Einschätzung derselben voraus. Erfasst werden anamnestische Angaben $\mathrm{zu}$ vorausgegangenen Unfällen und Behandlungen, geprüft die Symptome wie Schmerzen, Bewegungseinschränkung, Ergussbildung und Gelenkschnappen. Kniebandinstabilitäten und Achsfehler stellen bedeutende pathogenetische Faktoren dar und müssen auf jeden Fall separat beurteilt und adressiert werden.

Lokal müssen die Tiefenausdehnung der Defekte und die betroffene Fläche, die Lokalisation des lädierten Areals bezüglich seiner Belastungssituation und das betroffene Gelenkteil am medialen oder lateralen Femurkondylus, am Tibiaplateau, an der Patella oder im patellaren Gleitlager beurteilt werden. Dazu dient eine Arthroskopie, die in besonderen Fällen auch nur zu diagnostischen Zwecken durchgeführt werden kann. Der Tasthaken lässt als Indentor je nach Erfahrung des Untersuchers eine repoduzierbare Aussage über die elastischen Eigenschaften des Knorpels zu. Auch die Stabilität oder Instabilität der Ränder von Knorpeldefekten lassen sich damit zuverlässig beurteilen.

Ein großer Teil der Knorpelschäden wird während einer aus anderen Indikationen durchgeführten Kniespiegelung als einziger pathologische Befund oder als 


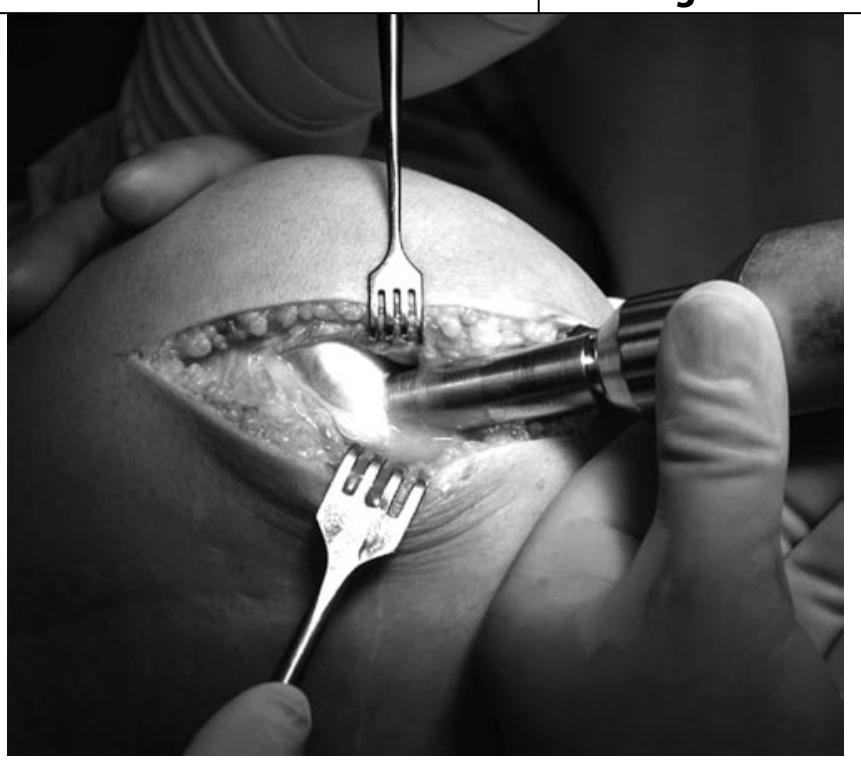

Abb. $1<\mathrm{DBCS}^{\oplus}$-Fräse während der Entnahme des Defektzylinders am medialen Femurkondylus

begleitender Zufallsbefund z. B. bei Meniskusresektionen festgestellt.

Bekannte Läsionen werden präoperativ einer magnetresonanztomographischen Diagnostik zugeführt. Viele Patienten mit Knorpelschäden werden vom niedergelassenen Arzt bereits mit entsprechenden Befunden vorgestellt. Hier können die bei tief greifenden Knorpelschäden oder bei Osteochondrosis dissecans über den Knorpel hinausgehenden subchondralen Schäden überzeugend beschrieben sein. Aufgrund der immer noch eingeschränkten Auflösung und ohne die Möglichkeit der mechanischen Manipulation, zudem ohne therapeutischen Ansatz, sind magnetresonanztomographische Befunde bis heute bei Knorpelschäden nie definitiv zu werten, sondern immer nur ergänzend oder orientierend.

\section{Behandlung}

Es gibt mehrere Ansätze zur Behandlung eines Knorpelschadens. Kleinere Defekte $(<1,0 \mathrm{~cm}$ im Durchmesser) werden mit nachvollziehbaren Ergebnissen durch das Microfracture-Verfahren behandelt.

Bei größeren Läsionen hat sich die Knorpel-Knochen-Transplantation [z. B. als so genannte Mosaikplastik oder OATS („osteochondral autograft transfer system“) mit gestanzten Zylindern oder mit gefrästen Einzelzylindern als $\mathrm{DBCS}^{\circledast}$ („diamond bone-cutting system“)Verfahren] bewährt.

Ansätze mit dem Transfer von aus Knorpelgewebe gezüchteten Bindege- webszellen in Gelenkdefekte mit unterschiedlichen Verfahren zur Fixierung der Zellen im Defektbezirk [ACT (autologe Chondrozytentransplantation), MACI (matrixgekoppelte Knorpeltransplantation)] sind seit einigen Jahren mit laufend aktualisierten unterschiedlichen Konzepten und z. T. interessanten klinischen Ergebnissen im Einsatz. Eine abschließende Empfehlung kann hier noch nicht gegeben werden. Alle an vergleichbaren Konzepten arbeitenden Gruppen sehen deutlichen weiteren Optimierungsbedarf, besonders bezüglich der allgemein langen Rehabilitation und Entlastungszeiten.

Diffuse Arthrosen gesamter Gelenke erfordern dagegen unverändert den radikalen Oberflächenersatz durch Endoprothetik. Da diese künstlichen Gelenke aber nur eine begrenzte Haltbarkeit haben, verbietet sich diese Therapie bei jüngeren $\mathrm{Pa}$ tienten, hier findet in Einzelfällen eher die Arthrodese ihre Anwendung.

\section{Unterstützende Techniken}

Die folgenden Maßnahmen sind nicht in der Lage, Knorpelwachstum zu stimulieren.

\section{Gelenkspülung (arthroskopische Lavage und Débridement)}

Eine der ältesten und einfachsten Therapiemöglichkeiten stellt die Gelenklavage dar. Jackson [10] beschrieb, dass durch das Spülen eines Gelenks die Entfernung von Entzündungsmediatoren und freien Knorpelstückchen gelingt. Hierdurch kann es zu einer temporären Schmerzreduktion kommen, die Gelenkschädigung selbst wird nicht behoben.

\section{Knorpel-Shaving}

Durch die Abtragung des verletzten und instabilen Knorpels soll es zu einer Beseitigung der mechanischen Behinderung und dadurch einer Verminderung der begleitenden Entzündungsreaktion kommen. Diese Maßnahme führt, ähnlich der Lavage, nur zu einer temporären Schmerzreduktion bzw. einer Bewegungsverbesserung. Allerdings kann sie auch eine gesteigerte Produktion von Entzündungsmediatoren und eine Nekrose des angrenzenden gesunden Knorpels zur Folge haben.

Ähnlich wie bei der Lavage wird auch durch das Shaving die Gelenkschädigung prinzipiell nicht behoben, sondern es werden nur deren Folgen vorübergehend gemindert.

\section{Subchondrale Stimulation}

Die Grundidee solcher Techniken sind die Stimulation von mesenchymalen Stammzellen und deren Einstrom in den Knorpeldefektbereich, wo sie unter bestimmten Regenerationsbedingungen in der Lage sind, zu Zellen der chondrogenen Reihe zu differenzieren und den Knorpeldefekt mit Knorpelersatzgewebe mechanisch adäquat zu füllen. Um dies zu erreichen, wird mit einem Instrument eine Läsion in die sonst undurchlässige subchondrale Lamelle gesetzt, durch die Blut und Zellen in den Defekt strömen können und die Basis von Granulationsgewebe darstellen, in dem u. a. mesenchymale Stammzellen enthalten sind. Unterstützt wird dieser Prozess von Wachstumsfaktoren. Diese Therapieansätze erfordern eine zeitlich begrenzte Entlastung der Gelenke, während derer eine Strukturierung des Ersatzgewebes stattfinden kann. Kissing lesions, Knorpeldefekte auf gegenüberliegenden Gelenkflächen (z. B. Schienbeinkopf und Femurkondylen) können mit diesen Verfahren nicht behandelt werden.

\section{Pridie-Bohrung}

Bei dieser nach ihrem Erstbeschreiber benannten Methode wird im Bereich des Knorpeldefekts bis in die subchondrale 
Spongiosa hinein gebohrt. Aufgrund der entstehenden Reibungshitze durch den Bohrer sollte dieses Verfahren nur noch in Ausnahmefällen Anwendung finden.

\section{Microfracture-Technik}

Mit einem geschwungenen Pfriem werden, im Rahmen einer Arthroskopie, in der Defektregion mehrere Löcher in die subchondrale Lamelle am Boden der Knorpeldefekte geschlagen, welche sich mit Fissurlinien („microfracture“) untereinander verbinden. Hieraus resultiert, anders als bei der punktuell wirksamen Pridie-Bohrung, eine flächige Regenerationszone.

Vorteilhaft ist die universelle Anwendbarkeit auch an schwer zugänglichen Gelenkbereichen aufgrund unterschiedlicher Kröpfung des Pfriems, der wieder verwendbar und daher kostenneutral ist. Das Regenerationsgewebe ist knorpelähnlich, in der zellulären und biochemischen $\mathrm{Zu}$ sammensetzung handelt es sich aber eher um Narbengewebe mit einem hohen Anteil an Kollagen 2.

\section{Knorpeltransplantation}

Aufgrund der grundsätzlich sehr geringen spontanen und der unbefriedigenden induzierten Regenerationsfähigkeit des Knorpels sowie der wenig befriedigenden Ergebnissen der oben genannten Therapien bei größeren Defekten kommen auch aufwändigere Methoden mit der Transplantation von Knorpelgewebe zum Einsatz, wobei nur auf subchondralem Knochengewebe fixierter Knorpel eine mechanisch sinnvolle Funktion in belasteten Gelenken übernehmen kann..

\section{Eigenes Vorgehen - autogene Knorpel-Knochen-Transplantation}

Bei diesem erstmalig von Lexer 1908 beschriebene Verfahren wird der defekte Knorpel durch autologe Knorpel-Knochen-Stanzen ersetzt.

Die Indikation für dieses Verfahren stellen höhergradige oder tief greifende Knorpeldefekte („full thickness“, Outerbridge-Grad III-IV) bzw. eine Osteochondrosis dissecans Grad 3 und 4 in Last tragenden Gelenkanteilen von $1-9 \mathrm{~cm}^{2}$ dar. Prinzipiell kann dieses Verfahren in je-

Trauma Berufskrankh 2009 · 11[Suppl 1]:49-54 DOI 10.1007/s10039-008-1461-2

(c) Springer Medizin Verlag 2008

\section{N.M. Meenen · J.-P. Petersen · P. Ueblacker}

\section{Osteochondrale Transplantation. Ein bewährtes Therapieverfahren für größere Knorpeldefekte an Knie und OSG}

\section{Zusammenfassung}

Die Aussage des englischen Chirurgen W. Hunter von 1743, dass Knorpelschäden sehr unangenehm sind, nie heilen und schwieriger zu behandeln sind als Knochenschäden, trifft auch heute noch zu. Es existiert nach wie vor kein allgemein und umfassend erfolgreiches Konzept, das zu einer vollständigen Ausheilung der Defekte mit uneingeschränkter Wiederaufnahme der sportlichen oder alltäglichen Betätigungen führt. Derzeit werden kleinere Defekte durch das Microfracture-Verfahren, größere Läsionen mittels Knorpel-Knochen-Transplantation behandelt, während bei diffusen Arthrosen gesamter Gelenke meist der radikale endoprothetische Oberflächenersatz bzw. bei jüngeren Patienten eher eine Arthrodese zum Einsatz kommen. Die autogene Knorpel-Knochen-Trans- plantation ist ein viel versprechendes Verfahren mit guten Resultaten, die u. a. darauf zurückzuführen sind, dass der Defekt durch Zylinder von hyalinem, gesundem Knorpel mit den idealtypischen histologischen und biomechanischen Eigenschaften des Originalknorpels ersetzt wird. Weitere Vorteile sind die im Vergleich zu anderen Verfahren der Knorpel-Knochen-Transplantation geringe Entnahmemorbidität sowie die sofort mögliche Mobilisierung und Vollbelastung. Zudem ist das Verfahren kostengünstig.

\section{Schlüsselwörter}

Knorpelschaden - Microfracture-Verfahren . Knorpel-Knochen-Transplantation . Endoprothetischer Oberflächenersatz . Autogene Knorpel-Knochen-Transplantation

\section{Osteochondral transplantation. A reliable method for large cartilaginous defects of the knee and ankle}

\section{Abstract}

The statement made by the English surgeon W. Hunter in 1743 that "ulcerated cartilage is a very troublesome disease, its cure is more difficult than that of carious bone and when destroyed, it is never recovered" still applies to a great extent today. There is still no generally and comprehensively successful concept capable of producing complete healing of the damage with unrestricted resumption of sports or daily activities. Currently, small defects are treated using the microfracture method, larger lesions using osteochondral transplantation, while diffuse osteoarthritis of the whole joint usually require radical endoprosthetic resurfacing or, in younger patients, preferably arthrodesis. Autoge- nous cartilage-bone transplantation is a very promising technique with good results due to the fact that the defect is replaced by hyaline cylinders of healthy cartilage with the ideal histological and biomechanical characteristics of the native cartilage. Further advantages of the cartilage-bone transplantation method in comparison to other methods include low donor site morbidity, as well as immediate load-bearing and mobility. And the procedure is cost-saving.

\section{Keywords}

Cartilage defect $\cdot$ Microfracture procedure . Osteochondral transplantation · Autologous cartilage-bone transplantation 


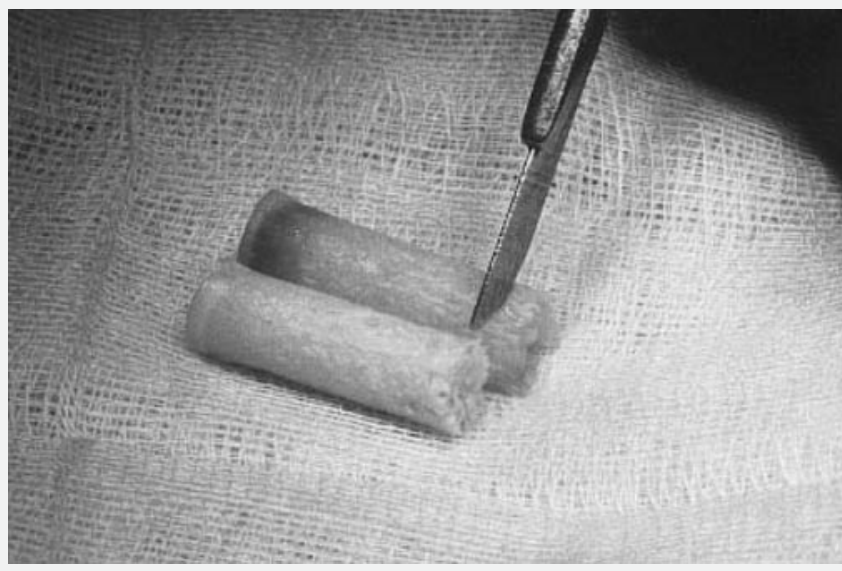

Abb. $2 \Delta$ Defektzylinder (vorne) und gut durchbluteter Transplantatzylinder (hinten) beim Ablängen vor der Implantation

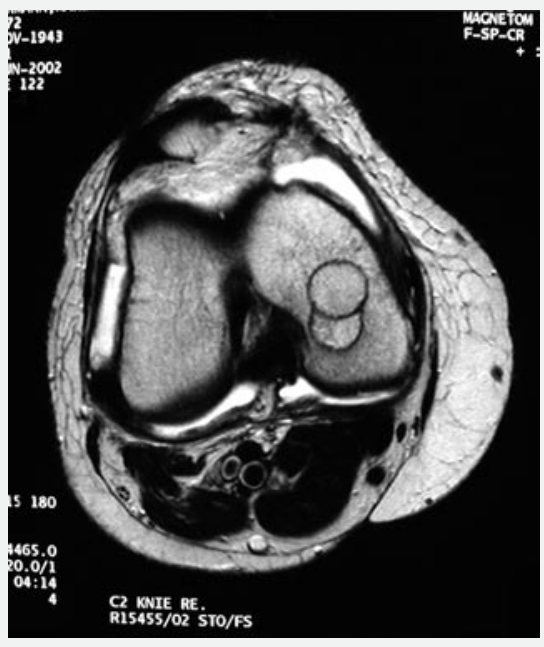

Abb. $4 \Delta$ Magnetresonanztomographie postoperativ mit 2 ineinander verzapften Transplantatzylindern

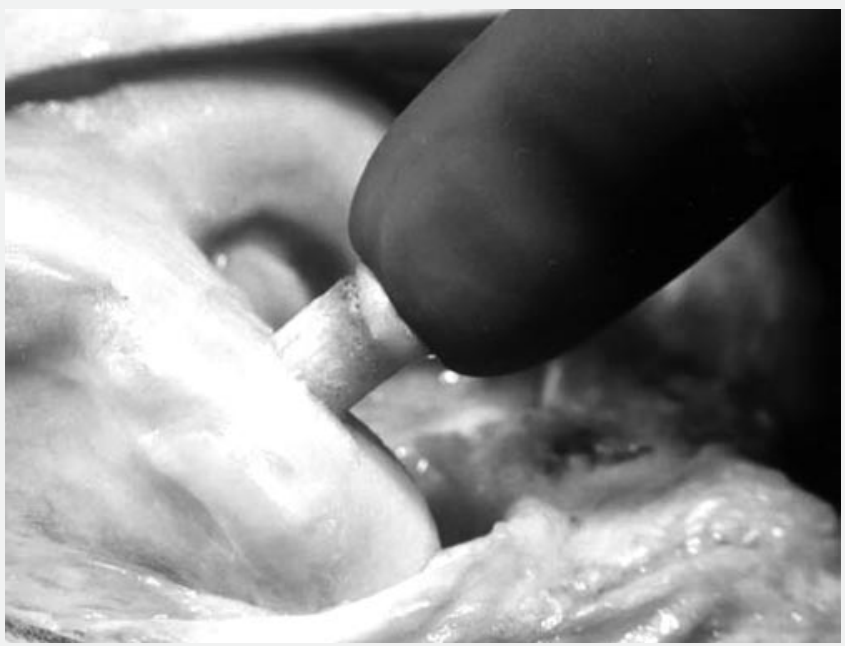

Abb. $3 \Delta$ Pressfit-Einbringen des Transplantatzylinders in den Defekt. Zur Schonung der Gelenkfläche darf das Transplantat nicht mit dem Hammer eingestößelt werden

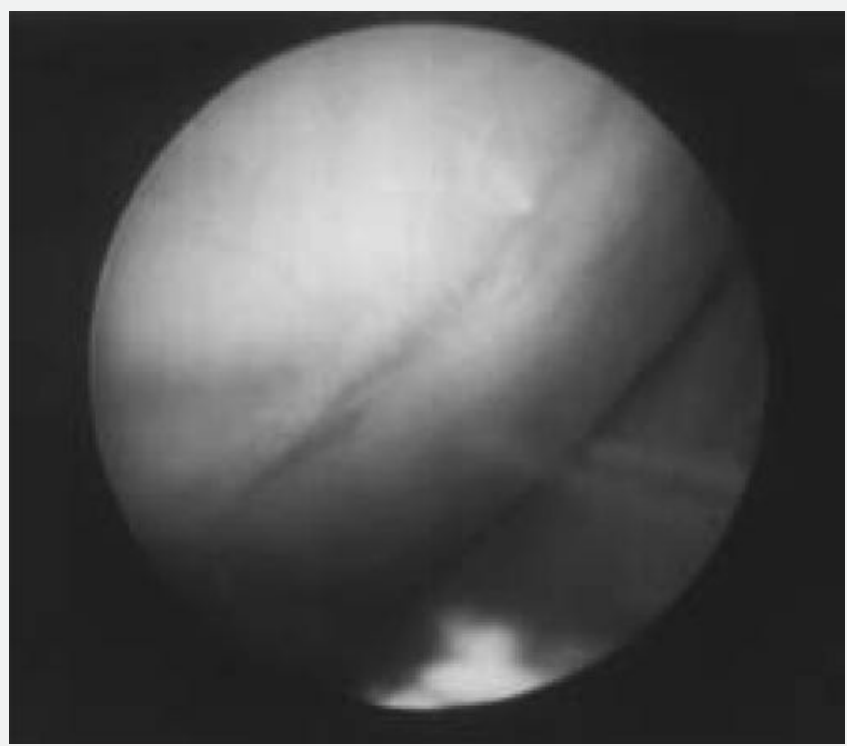

dem großen Gelenk angewendet werden. Etabliert haben sich hierfür allerdings die Femurkondylen des Kniegelenks und die Talusrolle am oberen Sprunggelenk. Hier finden sich auch am häufigsten Knorpeldefekte, die einer tief greifenden Behandlung bedürfen.

Bei dieser als offenes Operationsverfahren ausgelegten Therapie wird zunächst der Knorpeldefekt ausgemessen und mit einer Stanze oder Fräse mit einem 1,5 cm langen Spongiosazylinder ausgestanzt (• Abb. 1). Bei größeren und unrunden Defekten sind auch 2 oder mehr Zylinderfräsungen notwendig. Das so entstandene Defektareal wird im Anschluss mit einem autogenen Knorpel-KnochenZylinder gefüllt, der mit derselben Stanzoder Frästechnik entnommen wurde (• Abb. 2), und zwar aus einem wenig belasteten Teil des Kniegelenks, z. B. aus dem Randbereich zwischen dem patellaren Gleitlager und dem oberen Rand der Kondylenrolle, oder aus der Notch.

Die Knorpel-Knochen-Zylinder können mit unterschiedlichen Instrumentarien transplantiert werden. Wir verwenden für die Knorpel-Knochen-Transplantation das $\mathrm{DBCS}^{\circledR}$-Instrumentarium. Die Vorteile sehen wir in dem kühlen scharfen Schnitt im Knorpel und Knochen, den die diamantbesetzten und innen gekühlten
Fräsen setzen. Damit und mit den abgestuften Durchmessern der Zwillingsfräsen besteht die Voraussetzung für eine Pressfit-Implantation (- Abb.3) und damit für eine primäre Spongiosaheilung der Gewebezapfen aller verwendeten Fräsendurchmesser von 3-16 mm Durchmesser (• Abb. 4, 5). Die Fräsen haben eine gut ablesbare Tiefenskalierung, womit Defekt und Transplantat auch in der Länge des Knorpel-Knochen-Zylinders abgestimmt werden können (• Abb. 2).

Unsere histologischen Untersuchungen (- Abb. 6) zeigten bereits innerhalb von Tagen nach der Transplantation eine be- 
Abb. 6 Histologisches experimentelles Ergebnis 1 Woche nach Transplantation eines Knorpel-KnochenZylinders beim Minipig: Bereits beginnende knöcherne Integration (Pfeile) des subchondralen Knochens, Knorpel (Ausschnitt rechts) morphologisch intakt
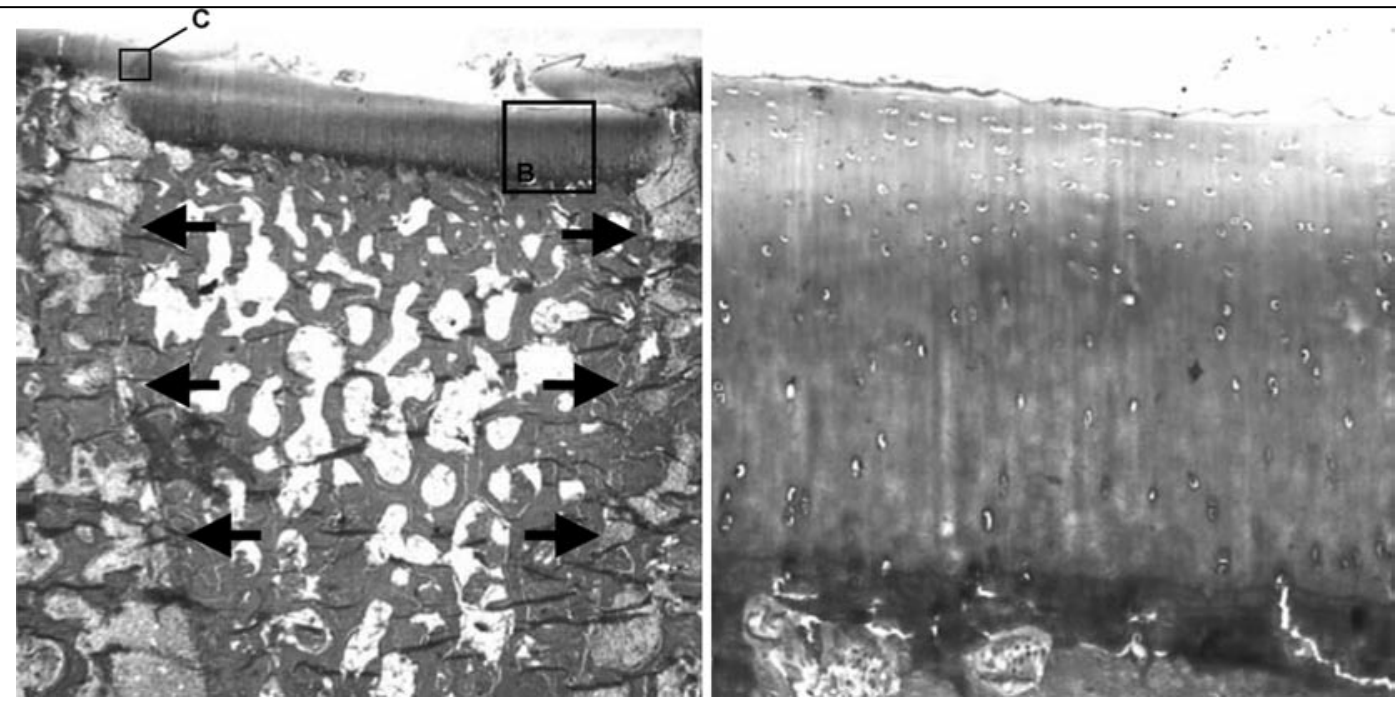

ginnende knöcherne Integration der Spongiosazylinder im Lagerknochen.

Der große Vorteil dieses Verfahrens ist, dass der Defekt durch Zylinder von hyalinem, gesundem Knorpel mit den idealtypischen histologischen und biomechanischen Eigenschaften des Originalknorpels ersetzt wird. Ein weiterer entscheidender Vorteil liegt darin, dass der Patient in der Nachbehandlung aufgrund der schnellen knöchernen Einheilung unmittelbar postoperativ voll belasten kann, ohne eine Einschränkung des Bewegungsumfangs. Dies ist sogar ausdrücklich gewünscht, da hierdurch der Sitz des Implantats verfestigt wird und es nicht zu einer langfristigen Mangelernährung und Degeneration des Knorpels kommt.

Die Entnahmestelle am patellaren Gleitlager wird von uns bei größeren Defekten ( $>9 \mathrm{~mm}$ Durchmesser) meist verschlossen. Dazu kann ein Teil eines längeren Transplantatzylinders oder ein ebenfalls mit $\mathrm{DBCS}^{\circledR}$ entnommener Knochenzylinder aus dem Beckenkamm dienen, der dort mit seinem Periostüberzug in minimalinvasiver Technik entnommen wird. Das Periost kann unter Gelenkbedingungen Faserknorpel bilden, wenn sich die Knochenoberfläche auf Niveau der subchondralen Lamelle befindet. Es können aber auch primär Zylinder von HAK (z. B. Endobon ${ }^{\circledR}$ ) eingepasst werden, denn wir konnten experimentell zeigen, dass Chondrozyten differenziert auf HAK wachsen können.

Auch von nicht aufgefüllten Entnahmedefekten konnten in unserem Patientengut keine negativen klinischen Folgen im patellaren Gleitlager festgestellt werden, die den positiven Gesamteffekt der Knorpel-Knochen-Transplantation für das Gelenk beeinträchtigt hätten.

Aber nicht nur das Instrumentarium unterscheidet sich von anderen Verfahren der Knorpel-Knochen-Transplantation. Der therapeutische Ansatz bei den DBCS $^{\circledR}$-Verfahren ist von dem Ziel einer vollständigen autogenen Gelenkflächenrekonstruktion geleitet. Beim OATS-Verfahren oder der Mosaikplastik werden zur Füllung des gleichen Defekts oft mehrere kleine Zylinder ausgestanzt, die nebeneinander implantiert werden. Dabei bleiben offene Spongiosaareale der spontanen Regeneration mit Faserknorpel überlassen. Die Integration der Implantate und die Auffüllung der periimplantären Defektzonen müssen abgewartet werden.

\section{Ergänzende operative Maßnahmen}

Sind die Knorpelschäden von relevanten Fehlstellungen verursacht oder begleitet, werden diese durch Umstellungsosteotomie korrigiert. Instabilitäten des vorderen Kreuzbands, die den Knorpeldefekt mitverursachen, werden zuvor oder gleichzeitig korrigiert. Meniskusrisse müssen äußerst sparsam reseziert oder refixiert werden.

\section{Nachbehandlungsschema}

Sehr früh können wir mit der Belastung beginnen. Passive Motorschienenbehandlung ergänzt die intensive Physiotherapie während der Rehabilitation. Wie erlauben
Sportlern spätestens nach 3 Monaten die Wiederaufnahme von Sport.

\section{Korrespondenzadresse}

\section{Prof. Dr. N.M. Meenen}

Pädiatrische Sportmedizin, Kinderorthopädie,

Altonaer Kinderkrankenhaus

Bleickenallee 38, 22763 Hamburg

norbert.meenen@kinderkrankenhaus.net

Interessenkonflikt. Der korrespondierende Autor gibt an, dass kein Interessenkonflikt besteht.

\section{Literatur}

1. Beaver RJ, Gross AE (1992) Fresh small-fragment osteochondral allografts in the knee joint. In: Aichroth PM, Cannon WD Jr (eds) Knee surgery, current practice. Deutscher Ärzteverlag, Köln, pp 464-471

2. Brittberg M, Lindahl A, Nilsson A (1994) Treatment of deep cartilage defects in the knee with autologous chondrocyte transplantation. N Engl J Med 331:889-895

3. Bruns J, Kersten P, Lierse W, Silbermann M (1992) Autologous rib perichondral grafts in experimentally induced osteochondral lesions in the sheepknee joint. Morphological results. Virchows Arch 421:1-8

4. Bulstra SK (1990) The potential of adult human perichondrium to form hyalin cartilage in vitro. J Orthop Res 8:328-335

5. Czitrom AA, Langer F, McKee N, Gross AE (1986) Bone and cartilage allotransplantation. A review of 14 years of research and clinical studies. Clin Orthop 208:141-145

6. Grande DA, Pitman MI, Petersen L (1989) The repair of experimentally produced defects in the rabbit articular cartilage by autologus chondrocyte implantation. J Orthop Res 7:208-218

7. Hangody L, Kish G, Karpati Z et al (1998) Mosaicplasty for the treament of articular cartilage defects: application in the clinical practice. Orthopedics 21(7):751-756

8. Homminga GN, Bulstra SK, Bouwmester PSM, Van der Linden AJ (1990) Perichondral grafting for cartilage lesions in the knee. J Bone Joint Surg Br 72 B:1003-1007 
9. Hunter W (1743) On the structure and diesease of articulating cartilage. Philos Trans R Soc 42:514521

10. Jackson RW (1991) Arthroscopic treatment of degenerative arthritis. In: McGinty JB (ed) Operative arthroscopy. Raven, New York, pp 319-323

11. Johnson LL (1996) Arthroscpic abrasion arthroplasty. In: McGinty JB (ed) Operative arthroscopy. Lippincott-Raven, Philadelphia, pp 427-446

12. Kato Y, Iwamoto M, Koike T (1987) Fibroblast growth factors stimulate colony formation of differentiated chondrocytes in soft agar. J Cell Physiol 133:491-498

13. Kreder HJ, Moran M, Keeley FW, Salter RB (1994) Biologic resurfacing of a major joint defect with cryopreserved allogenic periosteum under the influence of continous passive motion in a rabbit model. Clin Orthop 300:288-296

14. Lorentzon R, Hildigson C, Alfredson H (1996) Treatment of deep cartilage defects in the knee with periosteum transplantation. Abstract. Swedish Orthopaedic Association Meeting, Karlstad, Sweden

15. Magnusson PB (1941) Joint debridement. Surgical treatment of degenerative arthritis. Surg Gynecol Obstet 73:1-9

16. Malemud CJ (1993) The role of growth factors in cartilage metabolism. Osteoarthritis 19:569-580

17. Meenen NM, Rischke B (2003) Autogenous osteochondral transplantation (AOT) for cartilaginous defects of the femoral condyle. Operat Orthop Traumatol 15:38-56

18. Messner K, Maletius W (1996) The long term prognosis for severe damage to the weight-bearing cartilage in the knee. A 14-year clinical and radiological follow-up in 28 young athletes. Acta Orthop Scand 67:165-168

19. Mitchell N, Shepard N (1976) Healing of articular cartilage in intraarticular fractures of rabbits. J Bone Joint Surg Am 58:230-233

20. Oakeshott RD, Farine I, Pritzker KP et al (1988) A clinical and histological analysis of failed fresh osteochondral allografts. Clin Orthop 233:283-294

21. Petersen JP, Ruecker A, Stechow D von et al (2003) Present and future therapies of articular cartilage defects. Eur J Trauma 29:1-10

22. Petersen JP, Ueblacker P, Goepfert C et al (2008) Long term results after implantation of tissue engineered cartilage for the treatment of osteochondral lesions in a minipig model. J Mater Sci Mater Med 19(5):2029-2038

23. Pörtner R, Nagel-Heyer S, Goepfert C et al (2005) Bioreactor design for tissue engineering. J Biosci Bioeng 100(3):235-245

24. Pridie KH (1959) A method of resurfacing osteoarthritic knee joints. J Bone Joint Surg Br 41:618619

25. Ritsilae VA, Santavirta S, Alhopuro S et al (1994) Periosteal and perichondral grafting for cartilage lesions in the knee. Clin Orthop 302:259-265

26. Salter RB, Simmonds DF, Malcolm BW et al (1980) The biological effects of continous passive motion on the healing of full thickness defects in articular cartilage. An experimental investigation in the rabbit. J Bone Joint Surg Am 62:1232-1251

27. Stone KR, Walgenbach A (1997) Surgical technique for articular cartilage transplantation to full thickness cartilage defects in the knee joint. Operat Tech Orthop 7:305-311

28. Tippet JW (1991) Articular cartilage drilling and osteotomy in osteoarthritis of the knee. In: McGinty JB (ed) Operative arthroscopy. Raven, New York, pp 325-339
29. Vacanti CA, Kim WS, Schloo B et al (1994) Joint resurfacing with cartilage grown in situ from cell-polymer structures. Am J Sports Med 22:485-488

30. Wakitani S, Goto T, Pineda SJ et al (1994) Mesenchymal cell-based repair of large, full-thickness defects of articular cartilage. J Bone Joint Surg Am 76:579

31. Wagner H (1972) Möglichkeiten und klinische Erfahrungen mit der Knorpeltransplantation. Z Orthop Ihre Grenzgeb 110:714-715 